\title{
Creando contenidos curriculares digitales de Ciencias Sociales para Educación Primaria. Una experiencia TPACK para futuros docentes
}

\section{Creating digital curricular contents of Social Sciences for Primary Education. A TPACK experience for future teachers}

\author{
Ramón Cózar Gutiérrez*1 \\ ramon.cozar@uclm.es \\ JUAN ZAGALAZ* \\ juan.czagalaz@uclm.es \\ José Manuel Sáez López** \\ jmsaezlopez@edu.uned.es \\ * Universidad de Castilla-La Mancha, España \\ ** Universidad Nacional de Educación a Distancia, España
}

\section{Resumen:}

La introducción de las Tecnologías de la Información y de la comunicación (TIC) en las aulas ha generado una serie de cambios en los planteamientos del proceso de enseñanza-aprendizaje. Esto ha derivado en la aparición y desarrollo de numerosas tecnologías y pedagogías emergentes al servicio del docente en el aula. Se establece, por tanto, la necesidad de fomentar la capacitación de los docentes en TIC a través de la interacción de tres grandes dimensiones: disciplinar, pedagógica y tecnológica. El objetivo principal de este trabajo ha sido valorar las percepciones de un grupo de 82 alumnos de la asignatura

\begin{abstract}
:
The introduction of Information and Communication technologies (ICT) in the classrooms has generated a series of changes in the approaches to the teaching-learning process. This has evolved into the appearance and development of many technologies and emergent pedagogies at the service of the teacher. It is necessary to encourage teacher training on ICT through the interaction of three great dimensions: discipline, pedagogy and technology. The main objective of this paper is to evaluate the perception of a group of 82 students of the subject "New methodologies for the teaching of History", part of the Degree of
\end{abstract}

1 Dirección para correspondencia (correspondence address):

Ramón Cózar Gutiérrez. LabinTic. Laboratorio de integración de las TIC en el aula. Facultad de Educación. Universidad Castilla-La Mancha. Pza. Universidad, 3. 02071 Albacete (España). 
"Nuevas metodologías para la enseñanza de la Historia" del Grado de Maestro en Educación Primaria de la Facultad de Educación de Albacete, en la que, partiendo del modelo TPACK como planteamiento en la intervención, han tenido que crear sus propios materiales y contenidos digitales. La investigación desarrollada es descriptiva y ha seguido un modelo mixto, integrando características de los enfoques cuantitativo y cualitativo. La metodología de intervención se realizó desde un enfoque activo y dinámico, basada en el Aprendizaje Basado en Problemas y el modelo TPACK. Los resultados han mostrado valores altos sobre la organización de la asignatura, la metodología empleada, la calidad y la utilidad de las herramientas tecnológicas utilizadas, así como la conveniencia de potenciar la capacitación y perfeccionamiento en TIC para la creación y autoproducción de contenidos digitales en la formación inicial de maestros.

\section{Palabras clave:}

Contenidos digitales; Ciencias Sociales; Formación inicial; TIC; TPACK.
Teacher of Primary Education at Albacete, in which, from the TPACK model as an intervention approach, they had to create their own materials and digital contents. The research developed has been described, following a mixed model, including characteristics from qualitative and quantitative models. The methodology of intervention was applied from an active and dynamic approach, based on Learning Based in Problems and on the TPACK model. The results have shown high values on the organization of the subject, the methodology applied, the quality, and the usefulness of the technological tools used. The results also highlight the benefits of fostering teacher training on ICT aimed at creating self-production of digital contents in teacher initial training.

\section{Key words:}

Digital contents; Social Sciences; Teacher training; ICT; TPACK.

\section{Résumé :}

L'introduction des technologies de l'information et de la communication (TIC) dans la salle de classe a généré une série de changements dans les approches du processus d'enseignement - apprentissage. Cela a entraîné l'émergence et le développement de nombreuses technologies et nouvelles pédagogies au service de l'enseignant en classe. Donc, on sent le besoin de fomenter la formation des enseignants dans les TIC grâce notamment à l'interaction de trois grandes dimensions: disciplinaire, pédagogique et technologique. Le but principal de ce travail a été basé sur la valorisation des perceptions d’un groupe formé par 82 élèves qui étudient la matière "Nouvelles méthodologies pour l'enseignement de I'Histoire", appartenant aux études supérieurs pour devenir professeur d'éducation primaire dans la Faculté d'Éducation à Albacete. Dans cette faculté, on a commencé par le modèle TPACK comme l'approche d'intervention, et pour cette raison on a dû créer des matériaux digitaux propres. L'enquête qu'on a développé est de type descriptive et a suivi un modèle mixte. Ce modèle a intégré des caractéristiques appartenant aux approches quantitative et qualitative. La méthodologie d'intervention a été effectuée selon un approche actif et dynamique, basée dans l'apprentissage par problèmes (APP) et au modèle TPACK. Les résultats ont montré des valeurs élevés sur l'organisation de la matière, la méthodologie utilisée, la qualité et l'utilité des outils technologiques utilisées. Ainsi que la convenance ou propos de potentialiser et de favoriser la formation et perfectionnement des TIC, pour créer et produire soi-même des contenus digitaux dans la formation initiale des enseignants.

\section{Mots clés:}

Contenus digitales; Études Sociales; Formation intiale; TIC; TPACK. 
Fecha de recepción: 6-1-2015

Fecha de aceptación: 3-6-2015

\section{Introducción}

En los últimos años estamos asistiendo al desarrollo de gran cantidad de herramientas, materiales multimedia interactivos, recursos didácticos y plataformas educativas que junto con la aparición de nuevos conceptos metodológicos están ampliando notablemente el ecosistema pedagógico de la integración de las TIC en el aula (Adell \& Castañeda, 2013), permitiendo el desarrollo de numerosas habilidades cognitivas, así como la adquisición y consolidación de las competencias digitales básicas (Del Moral, 2009). Las pedagogías emergentes están surgiendo al calor de las tecnologías del mismo apellido y se están introduciendo en la realidad educativa, intentando aprovechar todo su potencial comunicativo, informacional, colaborativo, interactivo, creativo e innovador en el marco de una nueva cultura del aprendizaje impuesta por nuestra era virtual (Adell \& Castañeda, 2012).

El rol del maestro del siglo XXI o 2.0 se ha visto modificado considerablemente, adquiriendo un papel más dinámico en tanto facilitador de los aprendizajes, y no tanto de mero transmisor de contenidos (Del Moral \& Villalustre, 2010) y, por tanto, su formación debe ir encaminada tanto a lo instrumental, en cuanto al uso y manejo de las herramientas tecnológicas procedentes de la Web 2.0, como a la capacitación pedagógica y didáctica necesaria para emplear e integrar estos nuevos recursos en los procesos de enseñanza-aprendizaje (Antón \& Zubillaga, 2005).

Por tanto, nos encontramos con unas tecnologías que, junto a la generalización de las políticas educativas destinadas a incorporar las TIC en las aulas (Area, Alonso, Correa, Del Moral, De Pablos, Paredes, Peirats, Sanabria, San Martín \& Valverde, 2014), están creando las condiciones para que la digitalización de los contenidos y recursos educativos empiece a ser una realidad y comience a desplazar a los materiales didácticos tradicionales de la escuela del siglo XX: los libros de texto (Area, 2013).

En otros tiempos (no muy lejanos) el profesor era el principal acceso al conocimiento ya que era el único que poseía los contenidos educativos generados por las editoriales, en cuyas páginas se empaquetaba el conocimiento de las diferentes materias y asignaturas que el alumno debía asimilar. En contraste, actualmente casi todo el conocimiento aca- 
démico se encuentra en la red, y las diferentes herramientas tecnológicas han posibilitado la creación de nuevos contenidos que permiten un abanico más amplio de "creadores". En esta nueva situación, el rol del profesor ha cambiado, ya que ha pasado de ser únicamente experto en contenido y transmisor del mismo a convertirse en facilitador de contenidos, consultor de información, colaborador en grupo, proveedor de recursos, supervisor académico, consejero/orientador, diseñador de medios, investigador, tecnológico, organizador/administrador, facilitador de aprendizaje, moderador y tutor virtual, evaluador continuo (Cabero, sf, Gisbert, 2001, Goodyear, Salmon, Spector, Steeples \& Tickner, 2001).

Por todo ello, la sociedad demanda profesionales docentes bien formados que se sientan cómodos con las TIC y sean capaces de integrarlas en sus prácticas educativas habituales. Llama la atención que en la mayor parte de las universidades españolas con titulaciones de formación del profesorado se ha optado por eliminar de los planes de estudio asignaturas troncales de "Nuevas tecnologías aplicadas a la Educación", relegando la formación en TIC a optativas o a un tratamiento transversal a criterio de los docentes de unas pocas asignaturas. Lo que conlleva que los futuros maestros, nativos digitales, no las estén utilizando como un recurso didáctico habitual en sus actividades de enseñanza y aprendizaje y no estén desarrollando la competencia digital docente en los términos deseables (Cózar \& Roblizo, 2014; Roblizo \& Cózar, 2015).

Aprender, experimentar e innovar con el trabajo en red y en la red sobre recursos y materiales didácticos se convierte, en estos tiempos de globalización, en una necesidad vital para el actual y para el futuro docente (Tarraga, Peirats \& San Martín, 2013). En este sentido el objetivo de este trabajo es valorar las percepciones de 82 alumnos, futuros maestros de Educación Primaria, ante la experiencia formativa llevada a cabo en la asignatura "Nuevas metodologías para la enseñanza de la Historia" en la que partiendo del modelo "Tecnological PedAgocial Content Knowledge", conocido como TPACK, como planteamiento en la intervención, han tenido que crear sus propios materiales y contenidos digitales curriculares de calidad.

\section{Marco teórico}

En la Orden del Ministerio de Educación, Ciencia e Innovación 3857/2007 de 27 de Diciembre, por la que se establecen los requisitos 
para la verificación de los títulos universitarios oficiales que habilitan para el ejercicio de la profesión de Maestro en Educación Primaria, se recoge la importancia de desarrollar en los futuros maestros, la competencia digital docente (objetivo 11). A partir de esta normativa, desde el año 2008, se comenzaron a desarrollar las memorias de los títulos de Grado de Maestro en Educación Primaria por las distintas universidades. En nuestro caso, dentro del planteamiento verificado para su aplicación en la Facultad de Educación de Albacete de la Universidad de CastillaLa Mancha, se incluyó una mención de "Historia, cultura y patrimonio" en la que se ubica el contexto de aprendizaje y experimentación de esta investigación: la asignatura "Nuevas metodologías para la enseñanza de la Historia". Con un carácter de formación didáctico-práctica, pretende conseguir que los alumnos del Grado de Maestro en Educación Primaria conozcan algunas de las posibilidades que les ofrecen las TIC en la enseñanza/aprendizaje de la Historia a través de un gran número de herramientas de búsqueda, gestión y edición de la información y la autoproducción de contenidos curriculares digitales que puedan, posteriormente, aplicar en la enseñanza de esta materia con sus alumnos.

\section{Contenidos curriculares digitales}

La llegada de las Web 2.0 y 3.0 ha supuesto una transformación muy importante sobre el acceso a los contenidos. La Red no es ya sólo un banco de recursos, como lo era anteriormente, sino que, además, se ha convertido en una plataforma para poder crear y trabajar con ellos (Palomo, Ruiz \& Sánchez, 2008). En esta nueva versión de la web son los contenidos los que están robando el protagonismo a los aspectos tecnológicos o de diseño, por lo que son muchos los autores que acentúan la importancia de la variable "contenidos" en torno a la integración de las TIC, insistiendo en la necesidad de vincular la formación del profesorado en este sentido en torno a cuatro aspectos fundamentales: acceso universal, creación efectiva, recopilación y conexión de contenidos (De la Torre, 2006).

Ante este inevitable panorama se están creando las condiciones necesarias para que la digitalización de los contenidos curriculares se esté convirtiendo en una realidad en numerosos países, calibrando incluso las posibilidades de sustituir por completo a los libros de texto. Corea del Sur, por ejemplo, ha previsto sustituirlos por tablets y otros dispositivos 
electrónicos en el año 2015. Otra medida similar es la llevada a cabo en otros lugares como Shanghai o Estados Unidos, y que ha Ilegado a España y a nuestra comunidad con el Plan Escuela Extendida: mochila digital. Todas estas políticas justifican y avalan este cambio con argumentos variados: "preparar a los estudiantes en las competencias digitales para la cultura del siglo XXI, reducir costes económicos, evitar malformaciones y problemas de salud provocados por el sobrepeso de las mochilas cargadas de libros, innovar las metodologías de enseñanza, entre otros" (Area, 2013).

En consecuencia, es evidente que la creación de nuevos materiales didácticos en formato digital es un sector que está creciendo y crecerá de forma notable a corto y medio plazo. Las editoriales han reaccionado a la nueva y previsible demanda con la puesta en marcha de sendas plataformas educativas, desde las cuales, de forma más o menos velada, siguen ofertando su modelo de negocio sustentado sobre el pago de unas licencias sobre libros completos y por un tiempo determinado. Este es un aspecto que en la actualidad resulta un tanto contradictorio al ir contra la corriente predominante en la que los usuarios se han adaptado a comprar canciones y no discos completos.

Esta posición de las editoriales ha provocado que surjan otras iniciativas alternativas, apoyadas por docentes que no comparten la comercialización de la educación y que aprovechando las herramientas más útiles que brinda la Web 2.0, con sitios donde ni tan siquiera es necesario bajar e instalar ningún programa, crean sus contenidos y recursos educativos libres de licencias y los comparten a través de la red. Iniciativas que han ido evolucionando en función de la cantidad del material compartido y de las crecientes posibilidades que han surgido con la producción de nuevas herramientas y formatos de contenidos de software libre, a pesar de las controversias que rodean a esta tecnología (San Martín, Peirats \& Sales, 2008). Estos progresos han hecho surgir nuevas propuestas, digamos populares, armonizadas y comunicadas a través de las redes sociales, que ofrecen nuevas posibilidades y líneas de desarrollo en el ámbito docente. La Web 3.0 posibilita ordenar y clasificar los contenidos para que los dispositivos sean capaces de interpretarlos y tomar decisiones a través del vínculo de estos datos. En este sentido, en los últimos años ha aparecido la curación de contenidos, por la necesidad, impuesta ante la masificación de la información, de discernir los contenidos de calidad de aquellos que no lo son. Son muchos los docentes que no solo crean 
y comparten sus contenidos, sino que los trabajan reutilizándolos, seleccionando, relacionando y dotándolos de sentido al vincular los recursos sobre temas específicos para facilitar su acceso y fácil identificación por parte de la comunidad educativa (Rabajoli, 2012).

\section{Conocimiento Técnico Pedagógico del Contenido (TPACK)}

En la última década, junto a la fecunda producción de estándares de competencias TIC para docentes (UNESCO, Australia, Bélgica, Chile, Estados Unidos, Francia, Noruega), son también numerosos los estudios que abordan la formación y el perfeccionamiento del profesorado en TIC (Cabero, 2014; Fernández, 2007; Gallego, Gámiz \& Gutiérrez, 2010; Kirschner \& Davis, 2003; Llorente, 2008; Maroto, 2007; Martínez, 2008; Mcvee, Bailey \& Shanahan, 2008; Merma, 2008; Peirano \& Domínguez, 2008; Prendes, 2010; Prendes, Castañeda \& Gutiérrez, 2010; Prendes \& Gutiérrez, 2013; Raposo, Fuentes \& González, 2006; Reyes \& Piñero, 2009; Silva, Gros, Garrido \& Rodríguez, 2006; Tejedor \& GarcíaValcárcel, 2006; Tello \& Aguaded, 2009), y en todos ellos se incide en la necesidad de fomentar una adecuada formación inicial del profesorado en TIC mediante la interacción de diferentes dimensiones (instrumental, semiológica/estética, curricular, pragmática, psicológica, productora/diseñadora, seleccionadora/evaluadora, crítica, organizadora, actitudinal e investigadora). Como bien señala Cabero (2010: 43) "las tecnologías, independientemente de lo potentes que sean, son solamente instrumentos curriculares y, por tanto, su sentido, vida y efecto pedagógico vendrá de las relaciones que sepamos establecer con el resto de componentes del currículum, independientemente del nivel y acción formativa a la que nos refiramos". Cuestiones que cobran una mayor importancia en nuestro ámbito universitario (Sigalés, 2004). Las TIC no se deben convertir en el fin, sino en un medio para que los estudiantes aprendan los contenidos de las asignaturas. Por tanto, consideramos que la capacitación en TIC de los docentes debe ir encaminada hacia tres dimensiones: instrumental, didáctica y disciplinar.

En este sentido, el modelo TPACK (Technological Pedagogical Content Knowledge) popularizado por Mishra \& Koehler (2006) y Koehler \& Mishra (2008) se ajusta a la perfección a nuestro planteamiento. Este modelo parte del Pedagogical Content Knowledge (PCK) formulado por Shulman (1986) en el que se establece que los maestros deben poseer 
fundamentalmente conocimientos pedagógicos y disciplinares como elementos clave del proceso de enseñanza-aprendizaje. EI TPACK da respuesta al creciente interés de integrar la tecnología al servicio de los procesos formativos, generando una serie de interrelaciones e interacciones entre las tres fuentes primarias de conocimiento: disciplinar, pedagógico y tecnológico. Como señala Cabero (dir., 2014) el modelo sugiere que los docentes deben poseer un conocimiento tecnológico sobre cómo funcionan las TIC y las maneras de utilizarlas; un conocimiento pedagógico, respecto a cómo enseñar eficazmente; y un conocimiento sobre el contenido de la materia que debe enseñar.

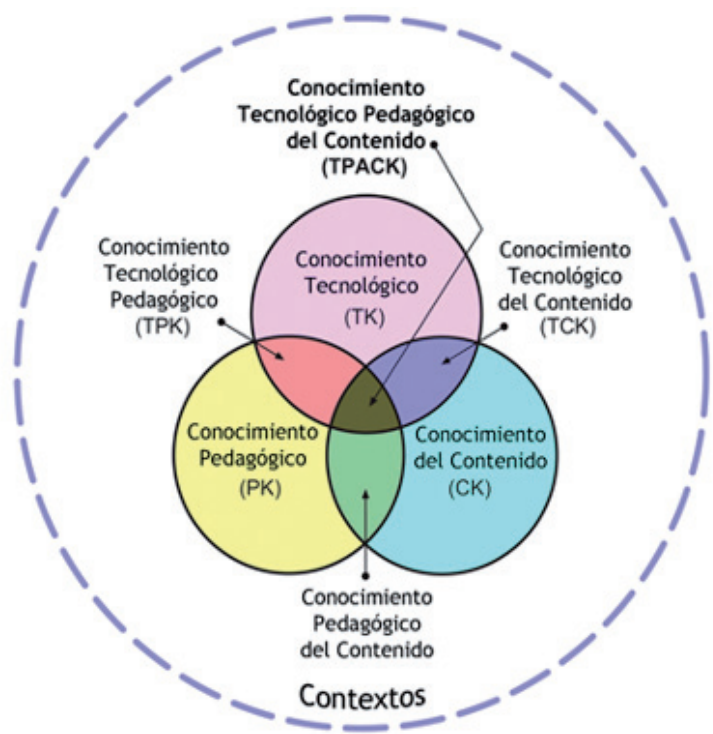

Figura 1. Modelo TPACK. Fuente: http://www.tpack.org/

Las interrelaciones e interacciones entre las dimensiones primarias dan lugar a otros tres tipos de conocimientos que surgen de la combinación de dos de ellas; y sólo cuando las tres confluyen se alcanza el objetivo del modelo: el conocimiento tecnológico pedagógico del contenido. Mishra \& Koehler (2006) sostienen que para generar una buena enseñanza con TIC se requiere comprender las interrelaciones entre cada uno de los tres elementos entre sí, para que, tomando en conjunto todas las intersecciones, el docente pueda desarrollar estrategias específicas y representaciones apropiadas para su contexto de trabajo (Manso, Garzón, Rodríguez \& Pérez, 2011). Pero se trata de un modelo flexible, que permite adaptar el modo en el que interactúan los tres tipos de conoci- 
miento a las circunstancias del nivel educativo y de la disciplina de la que se trate. De este modo, la integración de las TIC en el ámbito universitario requiere, no sólo un análisis de las distintas herramientas digitales que se pueden utilizar para la enseñanza, sino, además (y sobre todo) un análisis de las mismas enfocado a las disciplinas en las que van a ser empleadas y a las metodologías y técnicas docentes que vayan a integrar (Fernández López, 2012).

\section{Método}

\section{a) Enfoque y diseño}

La investigación que presentamos es descriptiva y para su desarrollo hemos seguido un modelo mixto, que incluye características de los enfoques cuantitativo y cualitativo (Tashakkori \& Teddlie, 2003), como estrategia exploratoria que nos permite tener una visión más amplia del fenómeno abordado. Respecto al enfoque cuantitativo, se utilizó un diseño no experimental descriptivo mediante encuesta, y para el cualitativo, un procedimiento de reducción de datos, categorización y codificación a partir de las respuestas a preguntas abiertas recogidas en el cuestionario.

\section{b) Intervención}

La experiencia formativa que vamos a analizar se ubica, como hemos comentado anteriormente, en el contexto de la Facultad de Educación de Albacete y se ha realizado durante tres cursos académicos (20122015), en el marco de la asignatura "Nuevas metodologías para la enseñanza de la Historia" de la mención de "Historia, cultura y patrimonio" del Grado de Maestro en Educación Primaria.

La metodología utilizada en esta asignatura se realizó desde un enfoque activo y dinámico basado en el Aprendizaje Basado en Problemas (Egido, Aranda, Cerrillo, De la Herrán, De Miguel, Gómez, Hernández, Izuzquiza, Murillo \& Pérez, 2006; Moust, Bouhuijs, \& Schmidt, 2007) y partiendo del modelo TPACK como planteamiento de intervención, para explorar la aplicación de diferentes recursos TIC con el fin de desarroIlar competencias, integrar los contenidos de Historia de forma amena y participativa y potenciar el aprendizaje significativo, experiencial, autó- 
nomo y colaborativo de los estudiantes. Se han trabajado recursos como wikis, foros, cómics digitales, webquest, PDi, HotPotatoes, Jclic, Realidad Aumentada, Neobook, videos educativos y gamificación.

En el proyecto dedicado a la creación de contenidos digitales educativos se emplea la herramienta que la Junta de Comunidades de CastillaLa Mancha, a través de su portal de educación, ha puesto a disposición de toda la comunidad, denominada Cuadernia (cuadernia.educa.jccm. es). Se trata de un software de autor con una interfaz y presentación final muy intuitiva, fácil de utilizar y con licencia Creative Commons que permite crear cuadernos digitales para utilizar en local o en red (Herrera, Gregori, Samblás, Sevilla, Montes \& Abellán, 2009).

Los estudiantes deben realizar un cuaderno digital que integre la digitalización de contenidos, actividades y evaluación de una unidad didáctica que desarrolle los contenidos mínimos establecidos en la legislación vigente sobre una de las etapas de la Historia. La intervención, tras una primera sesión en la que se desarrollan los aspectos "teóricos" y unas primeras orientaciones prácticas, se elabora de manera autónoma en grupos de tres-cuatro alumnos en cuatro sesiones, de dos horas cada una, a las que hay que añadir las horas de trabajo fuera del aula.

Al finalizar la asignatura se les aplicó un cuestionario a través de la plataforma campus virtual con la intención de recabar las opiniones y sensibilidades del alumnado ante los proyectos realizados y los recursos utilizados.

\section{c) Muestra}

La muestra del estudio, no probabilística e intencional, consta de 82 participantes que suponen el total de los estudiantes que han participado de la metodología por proyectos y del sistema de evaluación continua en la asignatura de "Nuevas metodologías para la enseñanza de la Historia" entre los años académicos 2012-2013 y 2014-2015. Distribuidos por cursos resultan tal y como aparece recogido en la tabla 1:

Tabla 1. Distribución de la muestra por curso académico y género.

\begin{tabular}{lccccc}
\hline CURSO & N & Varón & $\%$ & Mujer & $\%$ \\
\hline $2012-2013$ & 29 & 7 & 24.13 & 22 & 75.86 \\
$2013-2014$ & 40 & 14 & 35 & 26 & 65 \\
2014-2015 & 13 & 6 & 46.16 & 7 & 53.84 \\
TOTAL & 82 & 27 & 32.93 & 55 & 67.07 \\
\hline
\end{tabular}


Del total de participantes, el $67.07 \%$ eran mujeres y el $32.93 \%$ restante, de género masculino. La muestra es representativa de la disparidad de género que existe en los estudios de maestro donde hay una amplia superioridad de mujeres. La edad media de los estudiantes es de 22,86 años. El rango de edad va desde los 19 a los 44 años, si bien es cierto que el mayor porcentaje $(75,6 \%)$ tiene edades comprendidas entre los 20 y los 24 años. La distribución de la muestra por cursos no es homogénea, notando el descenso en cuanto al número de alumnos que se ha producido en el 2014-2015 debido a la reducción de plazas de nuevo ingreso y a la regulación de la homologación de las menciones con las antiguas especialidades. En cuanto al curso en el que están matriculados, el $40,24 \%$ son tercer curso, el $37,8 \%$ de cuarto y el $21,95 \%$ del curso de adaptación a los estudios de Grado.

\section{d) Instrumento}

La medición de las variables estudiadas se realizó a través de un cuestionario mixto, validado por expertos universitarios del grupo de investigación "Labintic. Laboratorio de integración de las TIC en el aula" de la UCLM. Este instrumento está encabezado por el apartado de datos socio-académicos (género, edad y curso) y dividido después en dos secciones: una serie inicial de cuestiones con cinco opciones de respuesta tipo Likert (de Muy en desacuerdo a Muy de acuerdo), en las que se pregunta al alumno su opinión sobre el uso de las TIC, la metodología y organización de la asignatura o los proyectos realizados y los recursos utilizados, entre otras; y se cierra con un apartado de respuesta abierta guiada, con el fin de recabar la opinión de los estudiantes sobre la utilización de estos recursos en las aulas, para recoger la información cualitativa. Para el análisis cuantitativo de los datos se han calculado estadísticos descriptivos (media y desviación típica) empleando para ello el paquete informático SPSS versión 19 para Windows. Para el análisis cualitativo, se categorizaron y codificaron las respuestas con el programa ATLAS-ti versión 6 para Windows.

Como coeficiente de fiabilidad para detallar la consistencia interna del aparato cuantitativo se empleó el análisis de los ítem mediante el Alpha de Cronbach, con un resultado de $\alpha=.81$, por lo que podemos considerar que su fiabilidad es aceptable (Bisquerra et al, 2004). 


\section{Resultados}

Los alumnos, jóvenes, en un 75\% entre los 20 y los 24 años, han crecido rodeados de tecnología y sujetos a sus rápidos y permanentes avances, por lo que no es de extrañar que su actitud hacia el uso de las TIC sea bastante favorable (Rodríguez, 2000). No aparecen los problemas de inseguridad o rechazo inicial frente a las TIC que se podían observar años atrás (Barnes, Marateo \& Ferris, 2007; Fainholc, 2008, Díez, Terrón, \& Anguita, 2009). A la pregunta sobre "Me gustan las nuevas tecnologías y las uso habitualmente" el 91\% se muestran "de acuerdo" o "muy de acuerdo" y el 100\% está "de acuerdo" o "muy de acuerdo" en que el uso de las TIC es favorable para la docencia.

Con respecto a la asignatura sus respuestas son muy positivas sobre su organización, la metodología docente empleada, la calidad y utilidad de las actividades realizadas o los materiales recomendados. Sobre el planteamiento de intervención, la interacción entre contenidos, pedagogía y tecnología, se realiza en todo momento de manera correcta y en coherencia con el modelo TPACK. El $100 \%$ de los encuestados contesta "de acuerdo" o "muy de acuerdo" a la pregunta sobre si está satisfecho de haber participado en una asignatura con esta metodología y un $97.5 \%$ también responde en esos mismos términos a si consideran que la organización de la asignatura ha sido una aportación positiva para su formación como maestro.

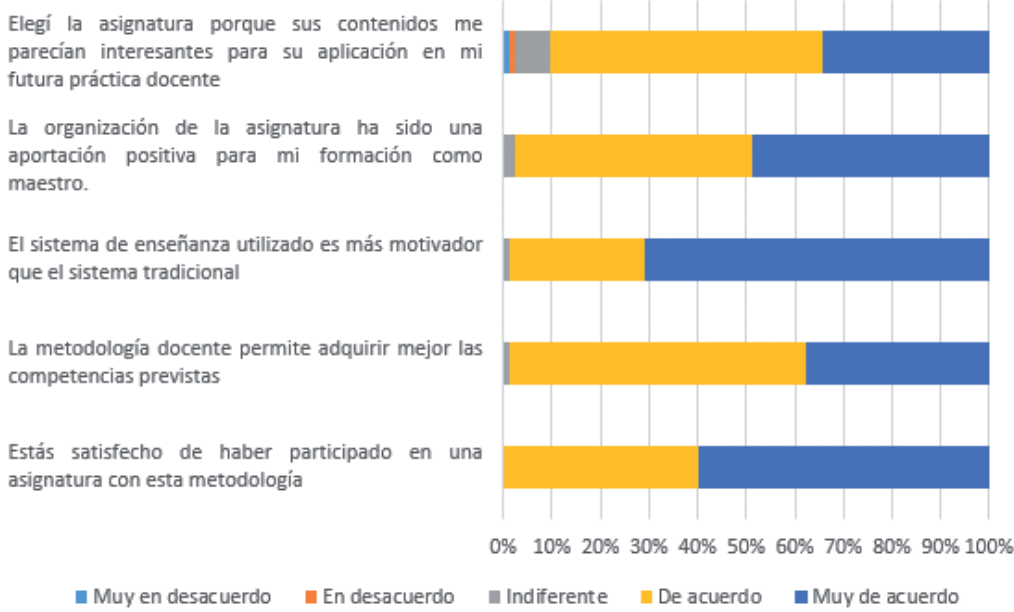

$0 \% \quad 10 \% \quad 20 \% 30 \%$ 40\% 50\% 60\% 70\% 80\% $90 \% 100 \%$

- Muy en desacuerdo $\quad$ En desacuerdo

घIndiferente $\quad$ De acuerdo $\quad$ Muy de acuerdo

Figura 2. Valoración sobre la organización y metodología de la asignatura. 
Estas valoraciones tan positivas así como la ausencia de dificultades a la hora de interrelacionar la tecnología con los contenidos curriculares de una materia como la Historia y sus particularidades para su enseñanza en Educación Primaria, también puede explicarse debido al carácter optativo en la elección de la asignatura, pues un 90\% de los estudiantes elige la asignatura porque sus contenidos le parecen interesantes para su aplicación en su futura práctica docente.

Tabla 2. Opiniones sobre los contenidos digitales educativos por curso académico.

\begin{tabular}{lllllllll}
\hline & 2012-2013 & \multicolumn{2}{l}{ 2013-2014 } & \multicolumn{2}{l}{ 2014-2015 } & Total & \\
\hline & Media & Dt. & Media & Dt. & Media & Dt. & Media & Dt. \\
\hline C1 & 3,55 & 0,99 & 3,55 & 0,96 & 3,85 & 0,99 & 3,60 & 0,97 \\
C2 & 4,48 & 0,57 & 4,43 & 0,50 & 4,62 & 0,51 & 4,48 & 0,53 \\
C3 & 3,93 & 0,80 & 3,98 & 0,58 & 4,46 & 0,78 & 4,04 & 0,71 \\
C4 & 4,45 & 0,51 & 4,53 & 0,55 & 4,46 & 0,52 & 4,49 & 0,53 \\
C5 & 4,48 & 0,51 & 4,53 & 0,51 & 4,54 & 0,52 & 4,51 & 0,50 \\
\hline
\end{tabular}

C1.- La creación propia de contenidos digitales educativos facilita un ritmo de trabajo más adecuado a mis disposiciones.

C2.- Considero que el uso de los contenidos digitales educativos puede resultar más motivador para los alumnos.

C3.- Los contenidos digitales educativos sustituiran a los libros de texto en papel.

C4.- Creando mis propios contenidos digitales educativos puedo conseguir que los alumnos los aprendan mejor.

C5.- El uso de contenidos digitales educativos permite que los alumnos adquieran mejor las competencias previstas,

En cuanto al análisis sobre las opiniones de los alumnos hacia la creación de contenidos digitales educativos, en la tabla 2 podemos ver los estadísticos descriptivos de las variables cuantitativas que hemos escogido para este artículo. La columna que resulta de más utilidad, al menos inicialmente, es la que indica las medias de las puntuaciones a cada uno de los ítems. Las desviaciones típicas (recogidas en la columna Dt) muestran unos valores que podemos considerar, dado el tamaño de la muestra, adecuados, porque se sitúan todos por debajo de un quinto del rango de la escala utilizada (Rowntree, 1981). Los valores de media más elevados se recogen en los ítem C5 [EI uso de contenidos educativos digitales permite que los alumnos adquieran mejor las competencias previstas] y C4 [Creando mis propios contenidos educativos digitales puedo conseguir que los alumnos los aprendan mejor], que inciden en la im- 
portancia que le otorgan a la creación de contenidos digitales educativos para favorecer la adquisición de competencias y sus posibilidades para una mejor comprensión de los contenidos. En los valores más bajos, rondando la categoría central de "Indiferente" aparecen los ítem C1 [La creación propia de contenidos digitales educativos facilita un ritmo de trabajo más adecuado a mis disponibilidades] y C3 [Los contenidos digitales educativos sustituirán a los libros de texto en papel]. La primera se puede explicar, como veremos más adelante, por los datos obtenidos en el análisis cualitativo, pues la falta de tiempo se observará como uno de las principales amenazas sobre la autoproducción de estos contenidos. Sobre la variable C3, si la analizamos por cursos académicos aparece una evolución favorable en cuanto a su consideración, fruto de la progresiva implantación de planes, programas y políticas educativas TIC de "mochilas digitales" que ha condicionado la opinión pública.

Tabla 3. Opiniones sobre la herramienta tecnológica Cuadernia.

\begin{tabular}{lllllllll}
\hline & \multicolumn{2}{l}{ 2012-2013 } & \multicolumn{2}{l}{ 2013-2014 } & \multicolumn{2}{l}{ 2014-2015 } & \multicolumn{2}{l}{ Total } \\
\hline & Media & Dt. & Media & Dt. & Media & Dt. & Media & Dt. \\
\hline T1 & 4,59 & 0,68 & 4,30 & 0,61 & 4,54 & 0,52 & 4,44 & 0,63 \\
T2 & 4,76 & 0,44 & 4,73 & 0,45 & 4,77 & 0,44 & 4,74 & 0,44 \\
T3 & 4,52 & 0,51 & 4,30 & 0,46 & 4,69 & 0,48 & 4,44 & 0,50 \\
T4 & 1,55 & 0,69 & 1,65 & 0,74 & 1,62 & 0,65 & 1,61 & 0,70 \\
T5 & 4,66 & 0,48 & 4,65 & 0,74 & 4,77 & 0,44 & 4,67 & 0,61 \\
T6 & 4,24 & 0,64 & 4,38 & 0,59 & 4,62 & 0,51 & 4,37 & 0,60 \\
T7 & 4,55 & 0,57 & 4,33 & 0,53 & 4,46 & 0,66 & 4,43 & 0,57 \\
T8 & 4,72 & 0,53 & 4,00 & 0,72 & 4,54 & 0,52 & 4,34 & 0,71 \\
\hline
\end{tabular}

T1.- Me ha parecido una herramienta tecnológica interesante.

T2.- Me ha resultado formativa.

T3.- Me permite plantear estrategias y contenidos innovadores que enriquecen notablemente el proceso de enseñanza/aprendizaje.

T4.- Es una pérdida de tiempo.

T5.- Aprender a utilizarlo mejora mis capacidades como maestro.

T6.- Utilizarlo facilita la comprensión de contenidos en el aula.

T7.- Manejarlo con soltura repercute en una mejora de los recursos educativos a la hora de impartir docencia.

T8.- Es fácil de usar.

También nos ha parecido interesante presentar la percepción del estudiante respecto al uso de la herramienta tecnológica utilizada para crear 
los contenidos digitales educativos. De nuevo recurrimos al análisis de los estadísticos descriptivos básicos. En la tabla 3 observamos cómo no sólo las medias de las preguntas que se formulan en positivo son elevadas, sino que también los datos presentan poca dispersión, lo que revela un elevado grado de confluencia en las puntuaciones de los alumnos y, por lo tanto, un mayor grado de consenso en torno a la valoración. Llama la atención como los ítem con una media más elevada son el T2 [Me ha resultado formativa] y el T5 [Aprender a utilizarlo mejora mis capacidades como maestro] por la vocación aplicada de los proyectos elaborados.

En cuanto al análisis cualitativo de los resultados, los datos obtenidos en las respuestas abiertas en las que los participantes tenían que dar su opinión sobre la utilización de contenidos digitales educativos en las aulas, se tradujeron en categorías con el fin de poder realizar comparaciones y posibles contrastes. Así, se establecieron dos categorías: fortalezas y debilidades. En la primera, el análisis y clasificación de las respuestas señalan por orden de frecuencia las dimensiones siguientes: su importancia como proyecto innovador se destaca en prácticamente todas las respuestas (95\%), seguido por su utilidad en su aplicación en una futura práctica docente $(69 \%)$ y su interés como propuesta motivadora (65\%), dinámica (65\%) y recomendable (53\%). En relación con la segunda categoría, tan solo un $30 \%$ de las respuestas recogen contenidos que podamos agrupar como debilidades. En estas se señalan también por orden de frecuencia, la carga de trabajo extra que puede suponer la autoproducción de contenidos digitales; relacionado, el tiempo que se le debe dedicar y, por último, la limitación de medios para su aplicación en las aulas. Respuestas que coinciden, del mismo modo, con lo aportado por otros estudios en los que se analizan las limitaciones del uso de las TIC en la Educación Superior (Ricoy \& Fernández, 2013).

A continuación recogemos algunas de las respuestas abiertas en las que se resaltan los valores positivos de esta experiencia didáctica:

H2_2012-13: "Creo que esta herramienta puede ser muy útil en la docencia a la hora de innovar y hacer tus propios contenidos. De este modo estás más liberado para escoger tus contenidos y no te ligas a lo que te dan las editoriales. Además de esto, los contenidos son de un modo más dinámico y práctico para los alumnos".

M22_2013-14: "Es una buena herramienta para llevar al aula, ya que en la actualidad los alumnos demandan el uso de las TIC, por lo que no tiene 
sentido que las utilicen en casa y se las prohibamos en el aula. Por ello, pienso que llevarlo a las aulas sería una buena opción que motivaría a los alumnos a aprender y querer saber más sobre los diversos temas que se tratan en el aula".

M6_2013-14: "Considero que el uso de las herramientas de autor para elaborar contenidos educativos digitales en el aula es bastante útil a la hora de realizar proyectos o materiales mucho más dinámicos. Esta herramienta nos permite crear nuestros propios contenidos acerca de un tema concreto, así como realizar una serie de actividades dinámicas para los niños, que hacen que tanto la asignatura de Historia, como cualquier otra se puedan explicar de una forma más amena, haciendo que el clima de aula sea lo más motivador y divertido posible"

M1_2014-2015: "Pienso que se debe convertir en una herramienta más de trabajo, ya que tiene muchas aplicaciones dentro de nuestro campo. Me ha gustado mucho y creo que se le puede sacar mucho partido dentro del aula".

\section{Discusión y conclusiones}

En los últimos años son numerosos los estudios que podemos encontrar en la literatura científica que abordan el estudio de las opiniones, percepciones y desarrollo de competencias para el uso de las TIC de los futuros maestros (Cabezas, Casillas \& Pinto, 2014; Gutiérrez, Palacios \& Torrego, 2010; Prendes, Castañeda \& Gutiérrez, 2010; Quintana, 2010; Raposo, Fuentes \& González, 2006; Roig \& Pascual, 2012; Ruiz, Anguita \& Jorrín, 2006; Suárez-Rodríguez, Almerich, Díaz-García \& FernándezPiqueras, 2012). En nuestro contexto más cercano destacan los trabajos de Cózar \& Roblizo (2014) y Roblizo \& Cózar (2015) en los que se pone de manifiesto ciertas carencias de los alumnos de cuarto curso del Grado de Maestro en Educación Primaria y Educación Infantil de la Facultad de Educación de Albacete en lo referente al manejo de recursos digitales educativos, entre otras habilidades TIC. Resultados que coinciden con los obtenidos en otros estudios (Rodríguez, 2000; Romero, Gisbert \& Carrera, 2009; Ruiz, Anguita \& Jorrín, 2006). Prendes, Castañeda \& Gutiérrez (2010) inciden en lo preocupante, en términos generales, que resultan estos mediocres resultados, pues se trata de futuros maestros, gran parte de cuyo trabajo pasará por la creación, evaluación y/o adaptación de recursos digitales a su práctica docente. 
Partiendo de la experiencia didáctica realizada con 82 alumnos, aprendices digitales (Esteve, Duch \& Gisbert, 2014) y futuros maestros, en la que han tenido que integrar su formación disciplinar, pedagogía y tecnológica mediante la creación y autoproducción de contenidos curriculares con una herramienta tecnológica como Cuadernia, los resultados nos muestran las posibilidades tan favorables que estos procesos ofrecen para desplazar los medios y recursos tradicionales y reinventar la escuela en los términos que expone Area (2012) al facilitar nuevas prácticas educativas que por una parte potencien el aprendizaje significativo, experiencial, autónomo y colaborativo de los estudiantes, y que por otra, desarrollen metodologías didácticas innovadoras que apuesten más por aprender a través de la elaboración de proyectos de investigación de problemas relevantes que por la repetición de lo que dicen los libros de texto. Los resultados muestran valores considerablemente altos sobre la organización de la asignatura, la metodología docente empleada, la calidad y utilidad de las actividades realizadas o los materiales recomendados. También son altamente positivas las valoraciones hacia la creación de contenidos digitales educativos y su integración en la realidad educativa y sobre el uso de la herramienta tecnológica utilizada para realizar dichos contenidos digitales en esta intervención.

Por todo ello, podemos concluir la conveniencia de potenciar la capacitación y perfeccionamiento en TIC en la formación inicial de los maestros siguiendo planteamientos como los establecidos en el modelo TPACK para que la integración de las TIC en las aulas se realice de manera coherente y real y no aislada e inconexa y se pueda dar respuesta a las necesidades demandadas por la sociedad de la información y el conocimiento.

\section{Referencias bibliográficas}

Adell, J. \& Castañeda, L. (2012). Tecnologías emergentes, ¿pedagogías emergentes? En J. Hernández, M. Pennesi, D. Sobrino y A. Vázquez (eds.). Tendencias emergentes en educación con TIC. Barcelona: Asociación Espiral, Educación y Tecnología (págs. 13-32).

Adell, J. \& Castañeda, L. (2013). El ecosistema pedagógico de los PLEs. En Castañeda, L. y Adell, J. (Eds.). Entornos personales de aprendizaje: claves para el ecosistema educativo en red. (pp. 29-52). Alcoy: Marfil.

Antón, P. \& Zubillaga, A. (2005): La formación del profesorado para la implantación de 
Creando contenidos curriculares digitales de Ciencias Sociales para Educación

Primaria. Una experiencia TPACK para futuros docentes

Ramón Cózar Gutiérrez, Juan Zagalaz y José Manuel Sáez López

las TICs como soporte a los nuevos modelos derivados del Espacio Europeo de Educación Superior (EEES), I Jornadas TIC. UNED. Madrid.

Area, M. (2012). La escuela del siglo XXI. Educación 3.0. 6

Area, M. (2013). De los libros de texto a los contenidos digitales: ¿cambio pedagógico o cambio del modelo de negocio? Disponible en http://www.relpe.org/especial-delmes/de-los-libros-de-texto-a-los-contenidos-digitales-cambio-pedagogico-o-cambiodel-modelo-de-negocio/

Area, M., Alonso, C., Correa, J.M., Del Moral, M.E., De Pablos, J., Paredes, J., Peirats, J., Sanabria, A.I., San Martín, A. \& Valverde, J. (2014). Las políticas educativas TIC en España después del Programa Escuela 2.0: las tendencias que emergen. Relatec. Revista Latinoamericana de Tecnología Educativa, 13(2), 11-33.

Barnes, K., Marateo, R.C. \& Ferris, S.P. (2007). "Teaching and learning with the net generation". Innovate: Journal of Online Education. 3(4).

Bisquerra, R. (coord.) (2004). Metodología de la investigación educativa. Madrid: La Muralla.

Cabero, J. (2010). Los retos de la integración de las TICs en los procesos educativos. Límites y posibilidades. Perspectiva educacional. Formación de Profesorado. 49(1), 32-61.

Cabero, J. (2014): "Formación del profesorado universitario en TIC. Aplicación del método Delphi para la selección de los contenidos formativos", en Educación XX1. 17(1), Madrid.

Cabero, J. (dir) (2014). La formación del profesorado en TIC: modelo TPACK. Sevilla: Universidad de Sevilla.

Cabero, J. (s/f): El rol del profesor en los nuevos entornos de comunicación. Disponible en http://reddigital.cnice.mec.es/1/cabero/01cabero_2.html

Cabezas, M., Casillas, S. \& Pinto, A.M. (2014). Percepción de los alumnos de Educación Primaria de la universidad de Salamanca sobre su competencia digital. EDUTEC. Revista Electrónica de Tecnología Educativa. 48, 1-14.

Cózar, R. \& Roblizo, M. (2014). "La competencia digital en la formación de los futuros maestros. Percepciones de los alumnos de los Grados de Maestro de la Facultad de Educación de Albacete", RELATEC. Revista Latinoamericana de Tecnología Educativa, 13(2), 119-133.

De la Torre, A. (2006). Web Educativa 2.0. Edutec. Revista electrónica de tecnología educativa, 20.

Del Moral, M.E. (2009). La escuela digital. En Exposición: La escuela y la formación de maestros en Asturias. Oviedo, Universidad de Oviedo.

Del Moral, M.E. \& Villalustre, L. (2010). Formación del Profesor 2.0: desarrollo de competencias tecnológicas para la escuela 2.0. Magister. Revista Miscelánea de Investigación, 23, 59-70.

Díez, E.J., Terrón, E. \& Anguita, R. (2009). Percepción de las mujeres sobre el «techo de cristal» en educación. Revista Interuniversitaria de Formación del Profesorado, 23(1), 19-32.

Egido, I., Aranda, R., Cerrillo, R., De la Herrán, A. De Miguel, S. Gómez, M., Hernández, R. Izuzquiza, D. Murillo, F.J. \& Pérez, M. (2006), "Aprendizaje basado en problemas (ABP). Estrategia metodológica y organizativa del currículum para la calidad de la 
Creando contenidos curriculares digitales de Ciencias Sociales para Educación

Primaria. Una experiencia TPACK para futuros docentes

Ramón Cózar Gutiérrez, Juan Zagalaz y José Manuel Sáez López

enseñanza en los estudios de Magisterio", Revista Interuniversitaria de Formación del Profesorado, 20(3), 137-149.

Esteve, F., Duch, J. \& Gisbert, M. (2014): Los aprendices digitales en la literatura científica: diseño y aplicación de una revisión sistemática entre 2001 y 2010. Pixel-Bit. 45, 9-21.

Fainholc, B. (2008). Modelo tecnológico en línea de aprendizaje electrónico mixto (o «blended-learning») para el desarrollo profesional docente de estudiantes en formación, con énfasis en el trabajo colaborativo virtual. Red: Revista de Educación a Distancia, 21.

Fernández López, M. (2012). Tres presupuestos para la efectiva integración de las TIC en la enseñanza universitaria. I Congreso Virtual Internacional sobre Innovación Pedagógica y Praxis Educativa INNOVAGOGíA 2012, Sevilla,

Fernández, M. (2007). ¿Contribuyen las TIC a hacer de los profesores mejores profesionales?: ¿Qué dicen los directivos escolares gallegos? Pixel Bit, Revista de Medios y Educación, 30, 5-15.

Gallego, M.J., Gámiz, V. \& Gutiérrez, E. (2010): El futuro docente ante las competencias en el uso de las tecnologías de la información y comunicación para enseñar. EDUTEC.Revista electrónica de tecnología educativa, 34, 1-18.

Gisbert, M. (2001): Nuevos roles para el profesorado en entornos digitales. Salinas, J. y Batista, A. (Coord.): Didáctica y Tecnología Educativa para una Universidad en un Mundo Digital. Universidad de Panamá. Facultad de Ciencias de la Educación

Goodyear, P., Salmon, G., Spector, J.M., Steeples, C. \& Tickner, S. (2001): Competences for online teachers: a special report, Educational Technology, Research and development. Wilson Education Abstracts.

Gutiérrez, A., Palacios, A. \& Torrego, L. (2010). La formación de los futuros maestros y la integración de las TIC en la educación: anatomía de un desencuentro. Revista de Educación, 352, 267-293.

Herrera, G., Gregori, C., Samblás, M., Sevilla, J., Montes, R., Abellán, R. (2009) Cuadernia, una herramienta multimedia para elaborar materiales didácticos. @tic. revista d'innovació educativa, 2

Kirschner, P. \& Davis, N. (2003). Pedagogic benchmarks for information and communications technology in teacher education. Technology, Pedagogy and Education, 12(1), 125-147.

Koehler, J. \& Mishra, P. (2008). Introducing Technological Pedagogical Knowledge. En AACTE (Eds.). The Handbook of Technological Pedagogical Content Knowledge for Educators. Routledge/Taylor \& Francis Group/American Association of Colleges of Teacher Education.

Llorente, M.C. (2008). Aspectos fundamentales de la formación del profesorado en TIC. Pixel-Bit. Revista de Medios y Educación, 31, 121-130.

Manso, M., Garzón, M., Rodríguez, C. \& Pérez, P. (2011). Contenidos educativos digitales que promueven la integración efectiva de las tecnologías de la información y comunicación. Digital Education Review. 19. 56-67.

Maroto, A. (2007). El uso de las nuevas tecnologías en el profesorado universitario. PixelBit. Revista de Medios y Educación, 39, 211-223. 
Creando contenidos curriculares digitales de Ciencias Sociales para Educación

Primaria. Una experiencia TPACK para futuros docentes

Ramón Cózar Gutiérrez, Juan Zagalaz y José Manuel Sáez López

Martínez, J. (2008). Las condiciones institucionales de formación de los maestros para el uso de las nuevas tecnologías en la escuela primaria. EDUTEC. Revista electrónica de tecnología educativa, 27.

Mcvee, M. Bailey, N. \& Shanahan, L. (2008). Teachers and teacher educators learning from new literacies and new technologies. Teaching Education, 19(3), 197 210.

Merma, G. (2008). Competencias del profesorado para el uso de las tecnologías de la información y la comunicación en la enseñanza, en el marco del Espacio Europeo de Educación Superior. R. Roig. (dir). Investigación e innovación en el conocimiento educativo actual, Alcoy: Marfil 317-326.

Mishra, P. \& Koehler, J. (2006). Technological Pedagogical Content Knowledge. A new framework for teacher knowledge. Teachers College Record, 108(6), 1017-1054.

Moust, J.H.C., Bouhuijs, P.A.J. \& Schmidt, H.G. (2007). El aprendizaje basado en problemas: guía del estudiante. Cuenca, Ediciones de la UCLM.

Palomo, R., Ruiz, J. \& Sánchez, J. (2008): Enseñanza con TIC en el siglo XXI. La Escuela 2.0. MAD.

Peirano, C. \& Domínguez, M.P. (2008): Competencia en TIC: El mayor desafío para la evaluación y el entrenamiento docente en Chile. Revista Iberoamericana de Evaluación Educativa, 1(2) 106124.

Prendes, M.P. (dir) (2010). Competencias TIC para la docencia en la Universidad Pública española. Indicadores y propuestas para la definición de buenas prácticas. Programa de estudio y Análisis. Recuperado de http.//www.um.es/competenciastic

Prendes, M.P. \& Gutiérrez, I. (2013): Competencias tecnológicas del profesorado en las Universidades españolas. Revista de Educación, 361.

Prendes, M.P., Castañeda, L. \& Gutiérrez, I. (2010): Competencias para el uso de TIC de los futuros maestros. Comunicar, 35(18), 175-182.

Quintana, J. (2000): Competencias en tecnologías de la información del profesorado de Educación Infantil y Primaria. Revista Interuniversitaria de Tecnología Educativa, 0, 166-174.

Rabajoli, G. (2012). Recursos digitales para el aprendizaje: una estrategia para la innovación en tiempos de cambio. Webinar aprendizaje ubicuo. 14-16 de marzo. Recuperado de http://www.webinar.org.ar/sites/default/files/actividad/documentos/Graciela\%20rabajoli \%20Webinar2012.pdf

Raposo, M., Fuentes, E. \& González, M. (2006): Desarrollo de competencias tecnológicas en la formación inicial de maestros. Revista Latinoamericana de Tecnología Educativa, 5(2), 525-537.

Reyes, M. \& Piñero, R. (2009). La función de los medios tecnológicos en los nuevos planes de estudios de Magisterio. Pixel Bit, Revista de Medios y Educación, 33, 119-132.

Ricoy, M.C. \& Fernández, J. (2013), Contribuciones y controversias que genera el uso de las TIC en la Educación Superior: Un estudio de caso. Revista de Educación, 360, 509-532.

Roblizo, M. \& Cózar, R. (2015), “Usos y competencias en TIC en los futuros maestros de Educación Infantil y Primaria: hacia una alfabetización tecnológica real para docentes", Pixel Bit, Revista de Medios y Educación, 47, 23-39. 
Creando contenidos curriculares digitales de Ciencias Sociales para Educación

Primaria. Una experiencia TPACK para futuros docentes

Ramón Cózar Gutiérrez, Juan Zagalaz y José Manuel Sáez López

Rodríguez, F. (2000). Las actitudes del profesorado hacia la informática. Pixel-Bit: Revista de Medios y Educación, 15, 91-103.

Roig, R. \& Pascual, A.M. (2012): Las competencias digitales de los futuros docentes. Un análisis con estudiantes de Magisterio de Educación Infantil de la Universidad de Alicante. @tic. Revista d'innovació educativa. 9, 53-60

Romero, M., Gisbert, M. \& Carrera, F. X. (2009): Centro virtual de recursos de tecnología educativa: Una herramienta para la formación inicial de maestros en TIC. RUSC. Revista de Universidad y Sociedad del Conocimiento, 6(2).

Rowntree, D. (1981). Statistics without tears. London: Penguin.

Ruiz, I., Anguita, R., \& Jorrín, I. M. (2006): Un estudio de casos basado en el análisis de competencias para el nuevo maestro/a experto en nuevas tecnologías aplicadas a la educación. RELATEC: Revista Latinoamericana de Tecnología Educativa, 5(2), $357-$ 368.

San Martín, A., Peirats, J. \& Sales, C. (2008). Controversias en torno al software libre: practices y discursos en la docencia universitaria valenciana. Revista Iberoamericana de Educación a Distancia. 11, 135-156.

Shulman, L. (1986). Those who understand: Knowledge growth in teaching. Educational Researcher, 15(2), 4-14.

Sigalés, C. (2004). Formación universitaria y TIC: nuevos usos y nuevos roles. Revista Universidad y Sociedad del Conocimiento, 1(1), 1-6.

Silva, J. Gros, B., Garrido, J.M. \& Rodríguez, J. (2006): Estándares en tecnologías de la información y la comunicación para la formación inicial docente: situación actual y el caso chileno. Revista Iberoamericana de Educación, 38 (3).

Suárez-Rodríguez, J., Almerich, G., Díaz-García, I. \& Fernández-Piqueras, R. (2012): Competencias del profesorado en las TIC. Influencia de factores personales y contextuales. Universitas psychologica, 11(1), 293-309.

Tarraga, R., Peirats, J. \& San Martín, A. (2013). Docencia universitaria y TIC. Materiales en línea para la formación en Cmap Tools. Revista Fuentes, 13, 263-282.

Tashakkori, A. \& Teddlie, C. (eds.) (2003), Handbook of mixed methods in social and behavioral research, Thousand Oaks, Sage.

Tejedor, F.J. \& García-Valcárcel, A. (2006): Competencias de los profesores para el uso de las TIC en la enseñanza: análisis de sus conocimientos y actitudes. Revista Española de Pedagogía, 64 (233), 2143.

Tello, J. \& Aguaded, I. (2009): Desarrollo profesional docente ante los nuevos retos de las tecnologías de la información y la comunicación en los centros educativos. Pixel Bit, Revista de Medios y Educación, 34, 31-47. 
